Relations industrielles

Industrial Relations

\title{
Les lendemains de l'arrêt Port Arthur Shipbuilding
}

\section{Robert Gagnon et Pierre Verge}

Volume 25, numéro 2, 1970

URI : https://id.erudit.org/iderudit/028133ar

DOI : https://doi.org/10.7202/028133ar

Aller au sommaire du numéro

\section{Éditeur(s)}

Département des relations industrielles de l'Université Laval

ISSN

0034-379X (imprimé)

1703-8138 (numérique)

Découvrir la revue

Citer cet article

Gagnon, R. \& Verge, P. (1970). Les lendemains de l'arrêt Port Arthur Shipbuilding. Relations industrielles / Industrial Relations, 25(2), 349-368. https://doi.org/10.7202/028133ar
Résumé de l'article

Quelle a été l'attitude des arbitres depuis cet arrêt de la Cour suprême ${ }_{1}$ relatif à leurs pouvoirs en matière de contrôle de la sanction disciplinaire imposée par l'employeur ? L'ambivalence de leurs réactions pose à nouveau le problème de la portée véritable de cet arrêt.
Tous droits réservés (C Département des relations industrielles de l'Université Laval, 1970
Ce document est protégé par la loi sur le droit d'auteur. L'utilisation des services d'Érudit (y compris la reproduction) est assujettie à sa politique d'utilisation que vous pouvez consulter en ligne.

https://apropos.erudit.org/fr/usagers/politique-dutilisation/ 


\title{
Les lendemains de I'arrêt Port Arthur Shipbuilding
}

\author{
ROBERT GAGNON
}

PierRe Verge

Quelle a été l'attitude des arbitres depuis cet arrêt de la Cour suprême ${ }^{1}$, relatif à leurs pouvoirs en matière de contrôle de la sanction disciplinaire imposée par l'employeur? L'ambivalence de leurs réactions pose à nouveau le problème de la portée véritable de cet arrêt.

En l'absence d'une attribution spécifique de ce pouvoir dans la convention ${ }^{2}$, l'arbitre de griefs ne peut prétendre, à la suite de l'arrêt Port Arthur Shipbuilding (P.A.S.), faire office d'une instance générale d'appel de la décision de l'employeur en matière de discipline. Il n'exerce à cet égard qu'un pouvoir de contrôle, dont l'étendue précise est fonction du texte de la convention ${ }^{3}$.

Quelle est la portée réelle de cette distinction posée par l'arrêt? Nous tenterons de l'indiquer (I), à la lumière, notamment, de décisions subséquentes d'arbitres d'Ontario et du Québec, pour ensuite préciser les situations désormais régies, en pratique, par l'arrêt, son aire d'applicabilité (II).

GaGnON, Robert P., assistant à la faculté de droit de l'université Laval.

Verge, Pierre, professeur agrégé à la faculté de droit de l'université Laval.

1 Port Arthur Shipbuilding Co. v. Arthur, arrêt unanime de la Cour suprême du Canada, en date du 1er octobre 1968, rapporté à (1969) R.C.S. 85 (commentaire de R. Dussault et P. Verge à (1969) 24 Relations industrielles 199). Infirme un arrêt majoritaire de la Cour d'appel d'Ontario, Regina v. Arthurs, (1967) 2 O.R. 49, renversant lui-même le jugement de première instance, (1967)I O.R. 272. Décision du tribunal d'arbitrage, Re United Steelworkers and Port Arthur Shipbuilding Co., (1966) 17 Lab. Arb. Cas. 109.

2 Exemple, parmi beaucoup d'autres, d'une convention accordant plus de latitude à l'arbitre en regard de la sanction imposée par l'employeur: Bradley Brothers Ltd v. Métallurgistes unis d'Amérique (6230), (1969) R.D.T. 375, à la p. 379 (J. Lippé) - pouvoir, dans un cas de congédiement, notamment, de « rendre toute décision équitable dans les circonstances \$.

3 Arrêt P.A.S., Cour suprême, supra, note (1), notes du j. Judson, p. 90 : «It was the board that exceeded its authority in reviewing the decision of management by purporting to exercise a full appellate function $»$. 
LIMITES DU POUVOIR DE CONTRÔLE DE L'ARBITRE.

Les restrictions posées par l'arrêt tiennent, d'une part, à l'objet du contrôle de l'arbitre (A), à l'occasion d'une sanction disciplinaire et, d'autre part, aux conclusions auxquelles il peut arriver relativement à cette sanction (B).

\section{Objet du contrôle de l'arbitre.}

L'arbitre essentiellement est appelé à vérifier le motif du geste de l'employeur. Comme la convention le dit le plus souvent, il doit s'assurer de l'existence d'une «juste cause » à l'action de l'employeur. Depuis P.A.S., on peut s'interroger quant à la spécificité de ce motif ${ }^{\left(1^{\circ}\right)}$, à sa qualification, le cas échéant, selon la convention $\left(^{\circ}\right)$ et, finalement, quant à sa suffisance ${ }^{\left(3^{\circ}\right)}$.

\section{Spécificité du motif}

Le motif de l'employeur, dont l'arbitre est appelé à contrôler l'existence, doit-il être "spécifique », c'est-à-dire, approprié à la peine particulière imposée et apprécié en fonction de celle-ci ? Au contraire, ne doit-il être que "générique », en ce sens qu'il suffirait qu'il justifie l'imposition d'une sanction disciplinaire quelconque par l'employeur? Dans ce dernier cas, le contrôle de l'arbitre se limiterait à s'enquérir de ce que l'employeur a eu un motif d'imposer une mesure disciplinaire quelconque, abstraction faite de l'identité de la peine effectivement imposée par lui, dans l'espèce. Autant dire alors, sauf des situations d'injustice criante, que l'arbitre ne jouerait qu'un rôle passif.

De fait, certains arbitres, depuis P.A.S., ont cru devoir s'abstenir d'intervenir dès lors qu'il y avait matière à l'imposition d'une sanction quelconque 4 .

L'on s'appuie sur le fait que le juge Judson a nié au tribunal d'arbitrage, saisi de l'affaire P.A.S., le pouvoir de porter atteinte au congédiement imposé par l'employeur (aux trois salariés qui s'étaient absentés sans permission, pour aller travailler quelques jours chez un concurrent), après avoir énoncé que, dans l'espèce, la conduite des salariés constituait un motif à l'imposition d'une sanction (quelconque) :

«The sole issue in this case was whether the three employees left their jobs to work for someone else and whether this fact was a proper cause

4 Ainsi, Re Communication Workers of America and Northern Telephone Ltd., 19 Lab. Arb. Cas. 129 (E.E. Palmer, pres.); également, dans le même sens, $R e$ United Electrical Workers, local 512 and Trane Co. of Canada Ltd, 20 Lab. Arb. Cas. 107 (E.E. Palmer, pres.). A la p. 136 de la première décision, on lit: «(...) Clearly, the grievors had left themselves open to some disciplinary action and, by virtue of the holding in Port Arthur Shipbuilding case, this board cannot question, in the words of Judson, J., 'the particular for chosen' (en l'occurence, le congédiement, pour refus d'accepter certaines conditions d'hébergement). 
for discipline. Once the board had found that there were facts justifying discipline, the particular form chosen was not subject to review on arbitration $\gg 5$.

$\mathrm{Au}$ contraire, d'autres arbitres ont estimé de leur compétence de vérifier si l'employeur avait eu un motif spécifique, c'est-à-dire, par exemple, un motif de congédier ( les termes de la convention).

Ainsi, dans une affaire Sanguinet Automobile ${ }^{6}$, l'arbitre a écarté l'application de la décision de la Cour suprême dans la mesure où elle prétendait l'empêcher de vérifier l'existence d'une "proper cause », d'un motif adéquat, d'imposer une peine spécifique, à savoir le congédiment. Cette conclusion de la Cour suprême, si tel était bien le sens de sa décision, selon l'arbitre, "does not in any way flow from the facts of the Port Arthur case ». Elle ne saurait ainsi lier les arbitres :

«This latter case (Port Arthur) was based on a dismissal of the grievors, which is a very restricted and extreme form of discipline. This was the sole point at issue in that case, and the judgement of the Supreme Court should have stopped at that point. Instead however, the Court proceeded to establish a broad principle of law which with all due respect, went far beyond what was required to decide that case.

The Supreme Court has now broadened the legal principle flowing from - «dismissal for proper cause», which was created to justify the facts of the Port Arthur case, to include any disciplinary measure.

It is ... an obiter dictum, a gratuitous creation of a principle of law which did not necessarily flow from the Port Arthur Case and was not needed to decide it. There is a difference between a disciplinary measure and a discharge for proper cause. One is much broader and includes the other. Unfortunately, the Court has assimilated the two... $\gg 7$.

Il ne s'agit pas là d'un simple refus de l'arbitre d'appliquer une conclusion de la Cour suprême, mais bien plutôt, d'une tentative de sa part, de préciser, correctement, à notre point de vue, la portée véritable de l'arrêt ${ }^{8}$. Si la Cour avait posé - ce qui est loin d'être acquis, verronsnous - que le rôle de l'arbitre doit se borner à vérifier l'existence d'un motif à une sanction quelconque, sans égard à la nature de cette dernière, elle aurait posé, selon cet arbitre, une règle que l'on ne pourrait relier aux faits essentiels à partir desquels elle devait rendre jugement.

5 Arrêt P.A.S., supra note (1), à la p. 89.

6 Sanguinet Automobile Ltée v. Fraternité canadienne des cheminots, D.C.D., 520-16 (J.H. Lande, arb.)

7 Ibid., pp. 6 et 7.

8 Voir notamment A. L. Goodhart, «Determining the 'ration decidendi' of a case », (1930), 40 Yale L. J. 161; H. A. Hubbard, «Le processus judiciaire du Common law», (1968), R. du B. 1, part. aux pp. 15 et sq. 
Dans cette perspective, la cour devait donc conclure que le tribunal d'arbitrage pouvait contrôler l'existence d'un motif au congédiement ( «proper cause for dismissal »). Mais, précisément, deux indices portent à affirmer que, malgré ces passages précités des notes du juge Judson, ce dernier a bel et bien reconnu que le tribunal d'arbitrage avait le pouvoir de s'enquérir de l'existence d'un motif à un congédiement, peine spécifique (même si, par ailleurs, dans l'espèce, son rôle devait s'arrêter là).

D'une part, ainsi que l'a souligné l'arbitre dans l'affaire Sanguinet Automobile Ltée, le juge Judson de la Cour suprême affirme endosser la position qu'avait adoptée, en dissidence, le juge Schroeder de la Cour d'appel d'Ontario ${ }^{9}$. Or, ce dernier avait clairement énoncé :

«The board ought to have addressed its mind to but one issue, namely, as to whether or not the company had proper cause to discharge the grieving employees».

D'autre part, surtout, le juge Judson a lui-même clairement posé que, dans l'espèce dont la Cour suprême avait à décider, les salariés avaient donné à l'employeur un juste motif de congédiement ${ }^{10}$. Ce fait a d'ailleurs été souligné par le président du tribunal d'arbitrage dans une affaire Int'l Nickel of Canada Ltd :

«As pointed out in the reasons of Judson J. ... there was only one proper legal conclusion, namely, that the employees had given the management «proper cause for dismissal ». In the sentences preceeding that conclusion, His Lordship refers to the question whether there

9 Arrêt de la Cour d'appel d'Ont., Regina v. Arthurs, supra note (1), à la p. 55 (italiques ajoutés).

10 Arrêt de la C. suprême, supra, note (1), à la p. 89 : «Then there was only one proper legal conclusion, namely, that the employees had given the management proper cause for dismissal 》.

Compar. avec une décision d'arbitre antérieure, Nordair Pilots Association v. Nordair Ltée, (1965) R.D.T.65 (M. DuMesnil, arb.) L'arbitre (spec. p. 77), tout comme allait le faire le j. Judson, dans P.A.S., avait reconnu qu'en l'absence d'une disposition expresse de la convention lui donnant le pouvoir de modifier la sanction imposée par l'employeur, son rôle devait se limiter à déterminer si l'offense la justifiait ou non, sans tenir compte de «circonstances atténuantes» (p. 91). Cette position ferme de sa part se trouvait cependant énoncée à l'occasion, avec l'ambivalence dont devait faire montre le j. Judson dans sa terminologie ! À la page 77 :

\&I cannot, therefore, alter the disciplinary penalty or discharge because the agreement does not give me such a power. The primary point for me to determine is whether the offense is sufficient to warrant discharge. I cannot take into consideration good service record, seniority, or any other mitigating circumstances or give the benefit of the doubt. Once disciplinary action is justified, it is completely with the management's right to decide on the degree of the action to be used and I cannot review it and decide on a less severe disciplinary measure ». 
was « proper cause », but we consider it an unfair gloss on the Court's language to say that proper cause ( in the air», so to speak) is to be, simpliciter, the subject of determination. The cause must be proper or appropriate to the action taken $\gg 11$.

Il semble bien, en effet, que les parties à la convention avaient entendu que l'employeur ne puisse congédier que dans le cas où il aurait vraiment été en présence d'un motif justifiant le congédiement, peine en soi sévère, il faut en convenir.

L'on peut aussi observer, de façon générale cette fois, que la compétence de l'arbitre saisi d'un grief à la suite d'un congédiement, serait pratiquement inexistante, s'il devait se borner à vérifier l'existence d'un motif à l'imposition d'une sanction quelconque, puisqu'il devrait en quelque sorte entériner ce congédiement dès lors qu'il serait établi que l'employé a commis une faute pouvant justifier l'imposition d'une peine, même des plus bénignes.

En définitive, nous serions d'opinion qu'en dépit de ce que pourrait peut-être laisser penser une première lecture de certains passages, pris isolément, de la décision de la Cour suprême, la seule interprétation valable de cette dernière est à l'effet que, dans l'espèce, le tribunal d'arbitrage pouvait contrôler l'existence d'un motif («proper cause») à un congédiement, peine spécifique ${ }^{12}$.

\section{Qualification du motif}

L'emploi, dans les conventions collectives, de qualificatifs disparates, s'agissant du motif de l'imposition d'une sanction disciplinaire par l'employeur, est-il de nature à influer sur l'étendue du pouvoir du contrôle dont jouit l'arbitre à ce sujet? En d'autres termes, l'étendue de ce contrôle varie-t-elle selon que la convention qualifie le motif exigé de l'employeur de «motif (ou cause) juste », 《raisonnable », «suffisant », «approprié », ou autrement?

Dans l'affaire P.A.S., le jugement de première instance cassa la sentence arbitrale notamment au motif que les arbitres avaient parlé de « just cause » dans leur décision alors que la convention employait l'expression «proper cause ». Ces deux expressions, selon le juge, différaient de sens au point que leur confusion, par les arbitres, avait constitué une

11 Re United Steelworkers of America and Int'l Nickel Co. of Canada Ltd., 19 Lab. Arb. Cas 118, à la p. 121 (J.F.W. Weatherhill, pres.).

Dans le même sens, Re S.K.D. Manufacturing and lodge 890, International Association of Machinists, décision inédite, 26 juin 1969 (P.C. Weiler, pres.).

12 Dans Lee v. Compagnie de téléphone Bell du Canada, (1969) R.D.T. 176 (F. Morin, arb.), l'arbitre était en présence d'une clause à l'effet suivant : «La compagnie peut congédier ou suspendre un employé pour cause suffisante et raisonnable» (p. 177), il a alors défini de la même façon l'objet de son contrôle : «... les actes déclarés répréhensibles par l'employeur constituent-ils une 'cause suffisante et raisonnable' à cette décision de congédiement ? (p. 185)》. 
erreur de droit leur faisant perdre leur juridiction. Unanimement, la Cour d'appel d'Ontario et la Cour suprême du Canada ont écarté cette décision ${ }^{13}$.

Qu'en serait-il, cependant, si, cette fois, la convention disait simplement que l'employeur peut suspendre ou congédier un employé «pour cause $»$, sans plus?

Signalons simplement que la Cour d'appel du Québec, dans l'affaire Aluminum, a donné à cette seule expression «pour cause», le sens, dans l'espèce, de «cause valide de congédiement $» 14$.

Le tribunal d'arbitrage est maître de l'appréciation des faits que la preuve lui révèle. En l'occurrence, c'est à l'arbitre de décider si tel acte reproché au salarié par l'employeur a vraiment été posé par lui et s'il constitue, le cas échéant, un motif de congédiement. Normalement, le tribunal civil ne devrait donc pas se substituer à l'arbitre dans l'appréciation des faits d'un litige.

Pourtant, c'est précisément ce qu'a fait la Cour suprême dans l'affaire P.A.S. Malgré ce qu'on pourrait être porté à croire en lisant les notes du juge Judson lorsqu'il affirme que le tribunal d'arbitrage, dans l'espèce $« .$. determined not whether there had been proper cause, but

13 Arrêts P.A.S., supra note (1), à la p. 88 (C. suprême); aux pp. 54, 60, 68 et 69 (C. d'appel).

14 Aluminum Co. of Canada Ltd. v. Syndicat national des employés de l'aluminium d'Arvida Inc., (1966) B.R. 641 (commentaire de F. Morin, (1967) 22 Relations industrielles 116).

On a parfois cru que le juge Brossard en rendant cette décision unanime de la Cour d'appel du Québec, avait indiqué que, dès lors qu'une cause à l'imposition de quelque peine disciplinaire est établie, seule la méchanceté ou la malice peut vicier l'exercice du pouvoir disciplinaire de l'employeur. Nous soumettons que telle n'était pas la portée de la référence du juge Brossard à l'exercice malicieux ou méchant du pouvoir disciplinaire de l'employeur et, à cet effet, nous croyons utile de reproduire ce passage de ses notes :

«Quant au droit que l'employeur a de faire des distinctions entre divers cas d'espèce constituant tous des causes valides de congédiement, droit dont certains qualifient d'exercice de discrimination, c'est un droit inhérent au droit de congédiement pour cause; la discrimination qui n'est en soi que la faculté de distinguer ou de différencier n'a rien en soi d'illégal; manifestée, exprimée ou faite sous sa forme active dans l'exercice d'un droit contractuel, elle ne peut vicier cet exercice que si celui qui en use agit méchamment ou malicieusement ; »

(à la p. 652 ; italiques ajoutés).

Ainsi, on le voit, la remarque de la Cour quant à l'exercice malicieux ou méchant du pouvoir disciplinaire avait trait aux cas où déjà un motif valide de congédiement est acquis. Dans l'espèce, la convention collective énonçait simplement pourtant que l'employeur pouvait «rendre la discipline et congédier pour cause $\gg($ a. 18, p. 648). 
whether the company having proper cause, should have exercised the power of dismissal... ${ }^{15}$, les arbitres n'avaient pas conclu à l'existence d'une «proper cause » à un congédiement. En effet, tel que la Cour suprême le rapporte d'ailleurs elle-même subséquemment, les arbitres dirent : "We will hold that these breaches are not a proper cause for dismissal but call for suspension $\gg 16$.

C'est donc la Cour qui, renversant l'appréciation des arbitres, a posé que «there was only one proper legal conclusion, namely, that the employees had given management proper cause for dismissal $\gg 17$. (On remarquera qu'à cet égard le cas était différent de celui dont eut à décider la Cour d'appel du Québec dans l'affaire Aluminum. ${ }^{18}$ )

\section{Suffisance du motif}

En revisant ainsi au fond la sentence arbitrale, les juges de la Cour suprême devaient présupposer, à tort ou à raison, que les arbitres avaient excédé leurs pouvoirs, dans l'appréciation même de l'existence du motif ( «proper cause») au congédiement. On peut dès lors tenter d'établir quels sont, selon la Cour suprême, les limites du pouvoir d'appréciation des arbitres en la matière. En d'autres termes, selon quels facteurs les arbitres doivent-ils décider s'il y a motif à congédiement sans par là excéder leurs pouvoirs?

Globalement, on peut entrevoir trois types de facteurs auxquels l'arbitre pourrait éventuellement recourir dans l'exercice de son contrôle de l'existence du motif à la sanction spécifique imposée :

a) ... des facteurs se rapportant à l'infraction reprochée au salarié;

b) ... des facteurs se rapportant au contrevenant;

c) ... des facteurs se rapportant à l'employeur dans l'exercice de son pouvoir disciplinaire.

a) Facteurs se rattachant à l'infraction.

L'arbitre doit évidemment s'enquérir de la commission par le salarié de l'infraction qui lui est reprochée. Il doit constater l'existence ou

15 Arrêt P.A.S., supra, note (1), p. 89.

16 Ibid.

17 Ibid.

18 Aluminum Co. of Canada Ltd. v. Le syndicat national des employés de l'aluminium d'Arvida Inc., supra, note (14).

Rappelons que la Cour d'appel avait cassé la sentence de l'arbitre qui, après avoir reconnu que la gravité de l'offense justifiait le congédiement, avait substitué une suspension au congédiement, en se fondant sur le caractère discriminatoire de celui-ci, compte tenu des sanctions qui avaient été imposées par l'employeur à d'autres salariés coupables de la même infraction. Unanimement la Cour d'appel a alors décidé que dès qu'il est établi qu'il y a cause à congédiement, le jugement de l'arbitre doit s'arrêter et que toute autre conclusion de sa part dépasse sa compétence. 
l'absence de cette faute. Si son rôle s'arrêtait là, il devrait entériner la punition infligée par l'employeur dès lors que serait établie la matérialité de la faute du salarié. Mais, avons-nous vu, l'arrêt P.A.S. (à moins, bien entendu, qu'il ne s'agisse d'une convention qui restreigne le pouvoir de l'arbitre à ce simple constat ${ }^{19}$, reconnait, précisément, que l'arbitre doit s'interroger sur la réalisation du motif de la peine imposée. Ce faisant, l'arbitre doit donc comparer le poids de la faute du salarié à celui de la peine qui lui a été infligée, de façon à vérifier objectivement, l'existence d'une certaine adéquation ou proportionalité entre les deux.

Par ailleurs, on peut croire que l'appréciation même du poids de l'infraction puisse dépendre des circonstances immédiates dans lesquelles celle-ci a été commise. Même si la Cour suprême n'a pas abordé ce problème, rien, à notre avis, dans sa décision, n'interdit à l'arbitre la considération des circonstances immédiates de la commission d'un acte reproché au salarié. Il ne s'agit là, en effet, que de la recherche même du « motif », dont il appartient à l'arbitre de s'enquérir.

Egalement, l'arbitre peut tenir compte du fait que la convention prohibe, en termes plus ou moins exprès, le geste reproché au salarié. Précisément, verrons-nous ${ }^{20}$, la convention dans l'affaire P.A.S. faisait état, à tout le moins, d'une préoccupation de l'employeur quant au travail de ses salariés à l'extérieur ; n'y indiquait-on pas qu'aucune permission de s'absenter ne serait accordée à un salarié à cette fin $^{21}$ ?

Ce serait même là l'une des considérations qui auraient amené le juge Judson à dire que, dans l'espèce, le tribunal d'arbitrage n'avait d'autre choix que de poser que l'on n'était pas en présence d'une infraction bénigne, partant, qu'une peine sévère, en l'occurrence le congédiement choisi par l'employeur, s'imposait.

19 On peut, en effet, imaginer qu'exceptionnellement des conventions collectives restreignent expressément le pouvoir de l'arbitre au contrôle de la culpabilité ou de l'innocence du salarié à qui une faute est reprochée, laissant airsi toute latitude à l'employeur quant au choix de la sanction, une fois l'acte fautif prouvé. À titre d'exemple, semble-t-il, voir la clause de la convention collective qui était en vigueur dans l'affaire Syndicat national des employés municipaux de Dorval v. Cité de Dorval, D.C.D. 520-15 (J.P. Hurteau, arb.).

20 Voir infra p. 369.

21 Clause 11.03, reproduite à la p. 88 de l'arrêt P.A.S., supra, note (1). De plus, une clause 9.04 était à l'effet qu'une absence non motivée excédant cinq jours entraînerait la perte de l'ancienneté. 
Dans certaines circonstances, mais de façon généralement beaucoup moins contraignante, la mention de l'infraction dans le règlement d'entreprise pourrait également être un facteur pertinent ${ }^{22}$.

b) Facteurs se rattachant au contrevenant.

La vérification du motif de la sanction est la province usuelle de l'arbitre. Ce motif établi, l'arbitre ne peut verser dans le contrôle de l'opportunité de la peine.

Qu'en est-il à ce sujet de cette pratique de certains arbitres, à l'effet de tenir compte de certains facteurs se rattachant à la personne du salarié ? Ainsi en serait-il de sa bonne foi au moment de la commission de l'infraction ${ }^{23}$, d'un dossier favorable, de son ancienneté, ou d'autres facteurs du même ordre. L'arbitre a-t-il le pouvoir de se livrer à de telles considérations?

\section{Considérations atténuant la responsabilité.}

Ce point a été soulevé devant la Cour suprême dans l'affaire P.A.S.. Dans leur factum, les appelants ont en effet invoqué comme motif d'invalidité du jugement de la Cour d'appel d'Ontario, le fait que celle-ci avait décidé que le tribunal d'arbitrage n'avait pas excédé sa juridiction en donnant effet à des considérations telles l'ancienneté, leur bonne

22 Il y aurait lieu ici de distinguer la portée du règlement d'entreprise selon qu'il a fait ou non l'objet de négociations avec le syndicat.

Même dans le cas où il se présente comme une annexe à la convention, il pourrait arriver qu'il y ait lieu, selon l'arbitre, à donner préséance à certaines autres dispositions de la convention elle-même. Voir Regina v. McCulloch, exparte Dowty Equipment of Canada Ltd., infra, note (46).

S'il ne procède que du geste unilatéral de l'employeur, le règlement intérieur voit son importance relative décroître. Pour qu'on le retienne, il ne doit pas contredire la convention, être également, aux yeux de l'arbitre, clair, connu, et d'application continue. Ce dernier conserve toujours son pouvoir d'appréciation du motif de la sanction. Voir Re United Automobile Workers, local 1524 and General Spring Products Ltd., 19 Lab. Arb. Cas. 392 (J.F.W. Weatherhill); Re Lumber and Sawmill Workers Union, local 2537 and K.P.V. Co. Ltd., 16 Lab. Arb. Cas. 73 (J.J. B. Robinson). Exemple, maintenant, d'une situation où l'arbitre conclut à l'existence d'une sanction motivée, dans le cas d'une infraction à un règlement intérieur : Métallurgistes Unis d'Amérique (7114) v. Industries Valcartier Inc., D.C.D. 745-2 (J. Ferland).

23 Exemple : Métallurgistes-unis d'Amérique v. Industries Valcartier Inc., D.C.D. 745-3 (R. Lafrenière, arb.) La décision passe sous silence, toutefois, l'arrêt P.A.S. 
conduite antérieure «the work history, seniority, and hope of redemption of the three employees in question $\gg^{24}$.

Il nous semble que la décision de la Cour fasse droit à ce motif 25 . En effet, celle-ci a reproché aux arbitres de ne pas s'être limités à établir si l'employeur avait eu une juste cause de congédier les plaignants «but whether the company, having proper cause, should have exercised the power of dismissal » ${ }^{26}$. C'est donc, en quelque sorte, le «contrôle d'opportunité » de la sanction, exercé par le tribunal d'arbitrage, qu'ont condamné les juges.

A cet égard, le juge Judson dit souscrire aux propos du juge Schroeder dissident en Cour d'appel ${ }^{27}$. Or, le juge Schroeder avait retenu deux motifs pour casser la sentence arbitrale. Le premier était à l'effet, précisément, que le tribunal d'arbitrage avait erré en droit et excédé sa juridiction par une interprétation de la convention collective qui l'avait amené « to determine whether or not the company had acted reasonably or had exercised its discretion improperly in discharging the employees rather than merely suspending them $\gg 28$. Quant au second motif, il s'agissait de la considération par les arbitres, de l'ancienneté cles plaignants. motifs :

La conclusion du juge est significative quant au lien entre les deux

«In my opinion the erroneous construction placed by the board on the terms of the collective agreement led to an excess of juridiction in the exercise of its powers and to an error of law on the face of the award. Thus the award is bad on both grounds. If my interpretation of the agreement is valid, then the evidence as to the length of service of the offending employees was irrelevant and extraneous to the issue, hence wrongly admitted. Error in law invalidating the award can also be assigned on this latter ground $\gg 29$.

24 Factum de l'appelante, Cour suprême, dossier no. 10909, p. 6. Également, exemple récent d'une décision d'arbitre qui, sans mention de l'arrêt P.A.S., tenant compte notamment de l'ancienneté et de la fidélité d'un salarié, substitue une suspension à un congégiement, qui avait été imposé à ce dernier en raison d'une erreur dans l'exécution de son travail: Producteurs de sucre d'érable de Québec v. Syndicat catholique des producteurs de sucre d'érable de Québec, D.C.D., 510-4 (H. Lande, arb.). Au sujet de la possibilité de tenir compte, dans certains cas, de la réhabilitation possible du salarié, voir: United Automobile Workers, local 222 and Houdaille Industries, 17 Lab. Arb. Cas. (B.L. Adell).

$25 \mathrm{Ce}$ faisant, la Cour suprême a rejoint l'attitude adoptée antérieurement à cet égard, par la Cour d'appel d'Ontario dans Regina v. Bigelow, Exparte International Nickel Co., (1959) O.R. 527.

(L'on avait alors fait reproche au tribunal d'arbitrage, qui avait d'abord luimême posé que le salarié méritait amplement le congédiement, d'avoir tenu compte de divers facteurs relatifs à l'opportunité, dans l'espèce, de cette sanction.)

26 Arrêt P.A.S., Cour suprême, supra, note (1), à la p. 89.

27 Ibid.

28 Arrêt de la Cour d'appel, supra, note (1), p. 55.

29 Ibid., p. 57. 
Sous un autre angle, l'on peut donc dire que les arbitres, en tenant compte de facteurs tels que l'ancienneté, avaient en fait, selon cette opinion, exercé un contrôle d'opportunité de la décision de l'employeur et ainsi excédé leur juridiction. L'étude du dossier des plaignants était en somme l'expression concrète de ce contrôle d'opportunité, partant de l'excès de juridiction des arbitres.

(A l'opposé, les juges majoritaires de la Cour d'appel avaient décidé qu'en étudiant toutes les circonstances entourant la faute reprochée aux employés, le tribunal d'arbitrage avait agi dans les limites de sa compétence et que sa conclusion, à savoir qu'il y avait absence de motif valable de congédiement, devait être maintenue.)

Aussi, peut-on interpréter également comme une prohibition de tenir compte du dossier antérieur de l'employé, cette remarque du juge Judson :

«The sole issue in this case was whether the three employees left their jobs to work for someone else and whether the fact was a proper cause ... 》 30

La conclusion générale du juge, à l'effet que «the board... exceeded its authority in reviewing the decision of management by purporting to exercise a full appellate function ${ }^{31}$, conduit aussi à cette inférence.

Telle paraît être la position juridique adoptée par la Cour à ce sujet. Quant à son opportunité, compte tenu de la fonction que devrait idéalement exercer l'arbitre, il s'agit là d'une toute autre affaire ...

\section{Circonstances aggravantes.}

S'en suivrait-il, de façon symétrique, que l'arbitre ne puisse, à l'inverse, tenir compte d'éléments, cette fois, accablants pour le salarié ?

Par exemple, l'officier du syndicat doit-il une obligation de fidélité à la convention plus rigoureuse qu'un simple salarié ? ${ }^{32}$

Observons ici simplement, que l'employeur, qui aura lui-même fait valoir cet aspect, ne se plaindra pas de la sentence de l'arbitre qui en tiendrait subséquemment compte. Il en irait de même dans le cas où l'employeur se serait prévalu du mauvais dossier du salarié. Dans ce

30 Arrêt de la Cour suprême, supra, note (1), p. 89 (italiques ajoutés).

31 Ibid., p. 90.

32 Ainsi, le tribunal d'arbitrage dans P.A.S., supra, note (1) avait tenu compte du fait que l'un des trois salariés qui s'étaient absentés était le président du syndicat, pour lui imposer une suspension plus longue (à la p. 111). Voir cependant, à ce sujet : Re United Electrical Workers, local 504 and Canadian Westinghouse Co. Ltd., 10 Lab. Arb. Cas. 343. 
dernier cas, comme toujours, il reviendra toutefois à l'arbitre de s'assurer de la suffisance de la gravité de la dernière en date des infractions reprochées ${ }^{33}$.

c) Facteurs se rattachant à l'employeur.

La Cour suprême, entérinant la position adoptée par le juge Schroeder de la Cour d'appel d'Ontario, en dissidence, a nié au tribunal d'arbitrage le pouvoir de substituer son jugement à celui de l'employeur lorsque, tout au moins, il est établi que la sanction particulière - en l'occurence le congédiement - qu'il avait choisie était motivée, compte tenu de la convention collective. C'est dire que la seule question se rapportant à l'employeur que puisse se poser l'arbitre, demeure toujours * did management go beyond its rights ? \$34 — l'employeur a-t-il respecté la norme imposée par la convention en matière de discipline. Contrôle donc, seulement, de l'existence du fondement de la sanction, non de son opportunité.

Le motif existant, l'arbitre ne peut, selon l'arrêt, faire entrave à l'usage par l'employeur de sa discrétion. Un tempérament, à tout le moins, a déjà été entrevu dans un arrêt antérieur de la Cour d'appel du Québec : l'employeur peut vicier l'exercice de la discrétion qu'on lui reconnaît en l'utilisant de façon malicieuse ou méchante ${ }^{35}$.

33 Plus précisément, du moins selon la théorie arbitrale dite du «culminating incident $»$, la dernière en date des infractions doit elle-même justifier, en soi, l'imposition d'une sanction. La preuve d'infractions antérieures, d'une semblable nature et ayant normalement donné lieu à l'imposition de peines moindres, est admissible. pour établir le poids de la sanction occasionnée par la dernière infraction. Il ne s'agit pas pour autant de punir une seconde fois une infraction, ce qui est inadmissible, (voir infra, à la p. 364.) Voir notamment à ce sujet Re S.K.D. Manufacturing Limited, supra note (11), à la p. 12 ; Re United Automobile Workers, local 112 and Douglas Aircraft Co. of Canada Ltd, 18 Lab. Arb. Cas. 27 (H.W. Arthurs) ; Re Sudbury Mine, Mill and Smelter Workers and Falconbridge Nickel Mines Ltd, 16 Lab. Arb. Cas. 163 (J.C. Anderson, pres.). Également, Syndicat des ouvriers de la Régie des alcools du Québec v. Régie des Alcools du Québec, (1969) R.D.T. 385, à la p. 429 (J. Trépanier) ; Ville de Montréal v. Syndicat professionnel des ingénieurs de la Ville de Montréal, (1967) R.D.T. 513, à la p. 526 (J. Melançon) ; Re United Rubber Workers, Local 232 and Goodyear Tire and Rubber Co. of Canada Ltd, 17 Lab. Arb. Cas. 137 (J.A. Hanrahan, pres.).

34 Arrêt de la Cour suprême, supra, note (1), p. 90.

35 Aluminum Co. of Canada Ltd. v. Syndicat national des employés de l'aluminium d'Arvida Inc., supra, note (14).

La Cour d'appel a alors nié au tribunal d'arbitrage le pouvoir de casser la décision de congédiement de l'employeur au motif que, même s'il y avait eu cause établie de congédiement, ce dernier avait exercé une discrimination en ne congédiant que quelques-uns des employés ayant commis la même faute, faute justifiant objectivement cette peine du congédiement. À la p. $652:$ «... la discrimination qui n'est que la faculté de distinguer ou de différencier n'a rien en soi d'illégal; manifestée, exprimée ou faite sous sa forme active dans l'exercice d'un droit contractuel, elle ne peut vicier cet exercice que si celui qui en use agit méchamment ou malicieusement ; (..) 》 
Il y a place, ce nous semble du moins, pour d'autres exceptions. Ainsi en est-il, notamment de disparités entre les sanctions imposées aux auteurs de gestes répréhensibles, en substance identiques, à plus forte raison, aux co-auteurs d'une même infraction, en l'absence de circonstances atténuant ou aggravant la responsabilité relative de ces individus ${ }^{36}$.

Non seulement la sanction discriminatoire est-elle inadmissible ; il en va de même d'une dualité de sanction ${ }^{37}$, d'une sanction illégale ${ }^{38}$, ou encore, imposée en raison d'un acte sans rapport avec le contexte de la relation

36 Les notes du juge Brossard, dans l'arrêt Aluminum, ibidem, malgré le caractère restrictif de certains passages, semblent ailleurs laisser place à cette limitation, à l'exercice du pouvoir disciplinaire de l'employeur. La «discrimination », au sens péjoratif mise de côté, cette faculté de «différencier» ou de 《distinguer» dans l'imposition de peines, - que le juge Brossard considère comme étant de l'essence du pouvoir disciplinaire - ne se réduit-elle pas (p. 650) à la considération «... (du) degré et (des) circonstances de la participation de chacun aux actes illicites»? (Par exemple, comme dans l'espèce, la possibilité d'être plus sévère dans le cas de celui qui, en plus de participer à une action illégale, a incité d'autres à faire de même.)

Nous posons qu'en l'absence de telles circonstances aggravantes ou atténuantes particulières, l'arbitre ne peut, sans par là nier toute notion élémentaire de justice, laisser cours à l'imposition de peines différentes aux co-auteurs d'une même infraction, voire à des salariés qui ont commis des offenses, en substance, semblables. Voir à ce sujet : $\operatorname{Re}$ S.K.D. Manufacturing Limited, supra note (11), aux pp. 21 et 22. L'on y cite notamment Re Teamsters Int'l Union, local 880, and Thibodeau Express Ltd, 18 Lab. Arb. Cas. 28 (J.A. Hanrahan, pres.) ; Long Sault Yarns Ltd, décision inédite (C.H. Curtis), maintenue successivement, sans notes, par la Ont. H. Ct. (J. Lieff) et la Cour d'appel d'Ontario.

Voir également les décisions suivantes: Re United Automobile Workers and Canadian Car Fort William Division of Hawker Siddeley Canada Ltd, 19 Lab. Arb. Cas. 375 ; Re Int'l Ass'n of Machinists, lodge 1936 and Ladish Co. of Canada Ltd, 17 Lab. Arb. Cas. 168, (C.H. Curtis, pres.) ; Re United Automobile Workers, Local 1285 and American Motors (Canada) Ltd, 17 Lab. Arb. Cas. 158 (H. Krever, pres.) ; Re United Automobile Workers, Local 458 and Massey-Ferguson Ltd, 15 Lab. Arb. Cas. 284, (E.W. Cross).

37 Syndicat des ouvriers de la Régie des Alcools du Québec v. Régie des alcools du Québec, supra, note (33), à la p. 429 (J. Trépanier) ; également — mêmes parties et même arbitre, (1968) R.D.T. 513 à la p. 519 ; Métallurgistes unis d'Amérique (5186) v. Campbell Chibougamau Mines Limited, (1966) R.D.T. 412 (J. Bousquet) ; Re United Automobile Workers, local 707 and Ford Motors of Canada Ltd, 14 Lab. Arb. Cas. 303. (E.W. Cross); Re International Union of Electrical Workers and J.A. Wilson Lightning and Display Ltd, 8 Lab. Arb. Cas. 157 (R.S. Clark).

38 Quid, par exemple, d'un congédiement, sous prétexte que le salarié est sous le coup d'une saisie-arrêt ? Voir, consacrant l'illégalité de ce geste, l'a. 650 du Code de procédure civile.

( $\AA$ noter que dans le cas où l'on prétendrait qu'il y a eu congédiement, suspension ou rétrogradation pour activité syndicale, que le commissaire enquêteur serait seul compétent, à l'exclusion de l'arbitre, relativement à ce motif. Cossette v. Ludger Harvey et Fils Limitée, (1969) B.R. 91 (res.) - commentaire de F. Morin à (1968) 23 Relations industrielles 677. 
de travail ${ }^{39}$, ou même, sans que l'employeur n'ait pris ou pu prendre connaissance de motifs exculpatoires ${ }^{40}$.

Tel étant l'objet du contrôle de l'arbitre, de quelle latitude jouit-il, peut-on se demander, dans la formulation de ses conclusions?

\section{Conclusions de l'arbitre.}

Distinguons le cas où le motif de la sanction se vérifie (comme dans l'affaire P.A.S. ${ }^{\left(1^{\circ}\right)}$ de celui où, au contraire, il y a, de l'avis de l'arbitre, absence de motif ${ }^{\left({ }^{\circ}\right)}$.

\section{Le motif se réalise}

Ayant indiqué, ainsi que nous l'avons vu, l'objet du contrôle de l'arbitre en matière disciplinaire - compte tenu de la convention collective qui avait cours dans l'espèce - la Cour suprême en a tiré une conséquence très claire quant à la compétence même du tribunal d'arbitrage :

«Once the board had found that there were facts justifying discipline, the particular form chosen was not subject to review on arbitration... ... the board had no power to substitute its own judgement for that of management in the circumstances of this case $\gg 41$.

Ainsi, dès lors qu'il constate, prenant en considération la convention collective, que l'employeur avait le motif exigé de lui pour imposer la sanction particulière qu'il a choisie, l'arbitre doit mettre fin à son rôle en rejetant le grief et en endossant la décision de l'employeur. Il ne peut intervenir pour substituer, par exemple, au congédiement, une suspension qu'il jugerait, le cas échéant, personnellement plus opportune, ni, à plus forte raison, annuler simplement la décision de l'employeur.

\section{Absence de motif}

La même interdiction de substituer son jugement à celui de l'employeur s'imposerait-elle à l'arbitre qui, cette fois, conclurait que la peine particulière imposée par l'employeur l'a été sans le motif requis par la convention? L'arbitre, estimant, à titre d'exemple, qu'ill n'y a pas

39 Par exemple, selon les circonstances, une accusation - voire une condamnation pénale - peut n'affecter aucunement la qualité de la prestation de travail due par le salarié. Voir : Victoriaville Specialties Co. Ltd. v. Union int. des rembourreurs de l'A. du N., local 573, D.C.D. 547-1 (P. Lambert) ; Commission Hydro-Électrique de Québec v. Syndicat national de la construction de Hauterive, D.C.D. 572-2 (J. L. Péloquin) ; Re United Automobile Workers, local 1524 and General Spring Products Ltd, 19 Lab. Arb. Cas. 392 (J.F.W. Weatherhill).

40 Re United Brewery Workers, local 173 and Carling Brewery Ltd, 17 Lab. Arb. Cas. 138 (H.W. Arthurs).

41 Arrêt P.A.S., supra, note (1); à la p. 89. (L'expression «justifying discipline $\gg$ équivaudrait, selon nos propos antérieurs, à «justifying dismissal », dans l'espèce.) 
cause d'un congédiement, pourrait-il y substituer, s'il la croyait fondée, une suspension?

Tout d'abord, posons que l'on ne saurait s'autoriser de la décision P.A.S. pour affirmer que, même en l'absence de motif à la peine particulière imposée au salarié, l'arbitre n'a pas le pouvoir de substituer, s'il le juge nécessaire, une autre sanction à celle que l'employeur avait choisie, ou encore, simplement d'annuler cette sanction.

La Cour suprême elle-même a eu soin de laisser entrevoir des limites à l'application de la restriction qu'elle posait aux pouvoirs de l'arbitre : "The board had no power... in the circumstances of this case .... Or, nous le savons, les circonstances de cette affaire étaient telles qu'il y avait eu, du moins aux yeux de la Cour, une "juste cause de congédiement ». On ne peut présumer de la décision qu'aurait rendue celle-ci, si, au contraire, elle avait estimé que, dans l'espèce, il n'y avait pas eu cette "proper cause for dismissal». Sa décision est donc, selon nous, inapplicable à une telle hypothèse ${ }^{42}$.

Arrivé à la conclusion que le motif de la sanction n'existe pas, l'arbitre peut donc, en premier lieu, selon les circonstances, simplement casser la décision de l'employeur, partant, dans le cas d'un congédiement, ordonner la réintégration du salarié.

Ainsi en fut-il dans l'affaire Sanguinet Automobile, en exemple ${ }^{43}$. L'arbitre fait état d'une faute minime ne justifiant pas le congédiement ; il n'envisage cependant pas, peut-être à dessein, l'imposition d'une peine moindre.

Par contre, dans d'autres espèces, la conduite du salarié, si elle ne justifie pas la peine imposée, pourrait en appeler une autre, moindre, selon l'arbitre.

Dans l'affaire Lee, l'arbitre indiqua que, dans l'hypothèse où il en vient à conclure à l'absence de cause au congédiement, il « doit connaissant les circonstances particulières d'une affaire, substituer, s'il le juge nécessaire, la peine disciplinaire qui lui paraît suffisante et raisonnable mais qui se rapproche le plus de la peine maximum imposée par l'employeur \$44. A l'appui de cette solution, il explique qu'en se limitant à accueillir le grief, simplement, l'arbitre provoquerait « une série de situa-

42 Pour sa part, l'arbitre dans l'affaire S.K.D. Manufacturing, supra note (11), ne substitua une suspension à un congédiement, non justifié, qu'en faisant montre d'une certaine réserve quant à son pouvoir de le faire, à la suite de l'arrêt P.A.S.. Il s'appuya également sur une disposition particulière de la convention qui, selon lui, lui aurait accordé une certaine latitude à cet égard (à la p. 23).

43 Sanguinet Automobile Ltée v. Fraternité Canadienne des Cheminots, supra note (6).

44 Lee v. 'Compagnie de Téléphone Bell, supra, note (12), p. 185. Voir également la décision Producteurs de sucre d'érable de Québec, supra, note (24), où l'arbitre substitue également une suspension au congédiement. 
tions absurdes... où l'employeur imposerait une nouvelle sanction, le salarié formulerait un nouveau grief jusqu'à ce que l'arbitre ou le salarié lui-même soit satisfait de la decision de l'employeur. > Il conclut qu'il semble qu'une telle situation ne respecterait pas «l'économie du droit du travail $\gg$.

Cette économie exige, nous le savons, que les parties solutionnent leurs griefs efficacement et rapidement. A cet égard, il est du devoir de l'arbitre de disposer de façon complète et finale du litige qu'on lui soumet, pouvoir, d'ailleurs, que lui attribue expressément la loi ${ }^{45}$. Renvoyer les parties en laissant, le cas échéant, à l'employeur le choix d'une nouvelle sanction ne constituerait certes pas une solution efficace et à coup sûr dans bien des cas, ne règlerait le litige que partiellement et temporairement.

Il faut reconnaître que la pratique, antérieure à l'affaire P.A.S., à l'effet que l'arbitre, convaincu qu'il n'y a pas motif à congédiement, puisse substituer à celui-ci une autre peine, s'il le juge nécessaire, avait apporté des solutions plus satisfaisantes.

Bref, il résulte de la décision de la Cour suprême, que l'arbitre ne peut modifier la peine choisie par l'employeur dès l'instant où est établie l'existence d'une matière à l'imposition de cette peine spécifique. Particulièrement, s'il y a matière à congédiement, l'arbitre ne peut y substituer une suspension qu'il jugerait plus opportune. En revanche, l'arrêt ne peut s'appliquer à l'hypothèse contraire, où, cette fois, l'arbitre pose, dans les limites de sa compétence, qu'il n'y a pas matière à congédiement ${ }^{46}$.

Telles paraissant être les limites qui s'imposent à l'arbitre dans l'exercice de son pouvoir de contrôle de la décision de l'employeur, selon

45 Code du travail, S.R.Q., 1964, c. 141, a. 89.

46 Dans un arrêt Regina v. McCulloch, ex parte Dowty Equipment of Canada Ltd (1969) 5 D.L.R. (3d) 289, la Cour d'appel d'Ontario a refusé d'infirmer la décision d'un tribunal d'arbitrage qui avait substitué une suspension à un congédiement, dans le cas d'un employé qui s'était approprié du matériel appartenant à l'employeur, contrairement, notamment, au règlement intérieur, reproduit en annexe à la convention. ( $\mathrm{Ce}$ même règlement prévoyait d'ailleurs le congédiement comme sanction d'une première offense de cette nature.) La convention accordait cependant à l'arbitre le pouvoir de régler le grief, non seulement par le maintien de la sanction ou, au contraire, la réintégration du salarié, mais également «by any other arrangement which may be deemed just and equitable ».

Si le texte de la convention permettait d'ainsi disposer de l'espèce, la portée que le juge McGillivray reconnaîtrait à l'arrêt P.A.S. nous paraît trop large :

«the case in question is authority for the proposition that a board of arbitration in a discharge case has no power to vary the penalty of discharge imposed by management unless such authority is given in express terms by the agreement (p. 291)».

Une fois établi le motif du congédiement, faudrait-il préciser selon nous. 
l'arrêt P.A.S., précisons maintenant les situations que ce dernier est appelé à réagir.

\section{APPLICABILITÉ DE L'ARRÊT.}

L'on devrait d'abord ici se demander, de façon préliminaire, si l'arrêt rendu par la Cour suprême, à partir d'une affaire d'Ontario, est de nature à lier, strictement, les tribunaux du Québec ${ }^{47}$ et, par ricochet, les arbitres de cette province. Toutefois, en pratique, il faut convenir que la Cour suprême, instance finale pour le pays, cherchera à tout le moins, dans l'avenir, à être conséquente avec elle-même en pareille matière, la loi étant au même effet, indépendemment du lieu d'origine du litige. D'où, encore une fois, la nécessité d'établir la nature des situations qui devraient désormais être résolues selon l'arrêt.

A cet égard, l'on pourrait être tenté - à l'instar de certaines décisions d'arbitres - d'établir des distinctions basées, soit sur la nature de l'infraction (A), soit sur celle de la sanction (B).

\section{Nature de l'infraction}

La Cour suprême a, en définitive, conclu que le tribunal d'arbitrage avait outrepassé sa compétence, en prétendant mettre de côté le congédiement imposé par l'employeur à trois salariés qui s'étaient absentés pour aller travailler chez un concurrent. Concluant à l'erreur de droit de sa part, la Cour, a-t-on vu, nota (substituant sans doute indûment sa propre appréciation du fond à celle des arbitres - mais là n'est pas la question) : « these were not trivial breaches $\gg$ - il ne s'agit pas d'infrac-

47 Les lois du Québec et d'Ontario, dans la mesure où elles régissent l'arbitrage des griefs, ne paraissent pas comporter de divergences pertinentes. Il en serait évidemment autrement si l'on devait poser que le Code du travail, supra, note (45), accorde à l'arbitre des griefs, à l'instar d'un conseil d'arbitrage appelé à trancher un différend, le pouvoir de "rendre sentence selon l'équité et la bonne conscience». Rien ne s'opposerait alors, en raison de ce seul pouvoir, à ce qu'il puisse, dans les cas opportuns, agir comme l'avait fait, en exemple, le tribunal d'arbitrage dans l'affaire P.A.S.. Un arbitre, M. le juge Trépanier, a, à certaines occasions, exprimé l'avis que l'arbitre des griefs avait pouvoir de juger selon «l'équité et la bonne conscience ». Voir notamment, l'affaire Syndicat des ouvriers de la Régie des alcools, supra, note (33), à la p. 392. (considération de circonstances atténuantes et substitution d'une suspension à un congédiement).

Pour notre part, nous avons déjà fait état d'arguments à l'effet que l'on ne pouvait ainsi transposer à l'arbitre des griefs les pouvoirs du conseil d'arbitrage des différends : «Le forum de la convention collective», (1967-68) 9 C. de D. 563, à la p. 589, n. 68. Adde F. Morin et J. Dupont, Annotation et jurisprudence des lois du travail du Québec, Québec, Soc. ed. soc. et jurid., p. C.T./88/ 5. 
tions bénignes. La convention, on le sait, se préoccupait spécifiquement du travail à l'extérieur; elle était à l'effet qu'aucune permission de s'absenter ne serait accordée à cette fin ${ }^{48}$. S'ajoutait également cette disposition à l'effet que l'absence non motivée de plus de cinq jours pouvait entraîner la perte de l'ancienneté. A tout le moins, peut-on dire, les signataires de la convention s'étaient préoccupés à l'avance de la conduite reprochée aux salariés, même s'ils n'en avaient pas fait formellement une infraction. De là, sans doute, l'incitation pour le juge Judson à conclure à l'erreur de droit des arbitres; la gravité de l'infraction motivait un congédiement, contrairement à ce qu'avait décidé le tribunal d'arbitrage.

Par analogie, des arbitres ont subséquemment tenté de circonscrire ainsi la portée de l'arrêt : il ne régirait vraisemblablement que des situations d'inconduite grave ${ }^{49}$, ou encore, allant à l'encontre de dispositions expresses de la convention ${ }^{50}$, ou même seulement, des situations dans lesquelles ces deux éléments se retrouvent à la fois ${ }^{51}$.

En regard d'une peine spécifique, elle-même lourde, en l'occurrence, un congédiement, poser ainsi la nécessité d'un manquement grave de la part du salarié recoupe forcément cette nécessité d'un motif adéquat (ou proportionné) à son imposition, nécessité, avons-nous établi, retenue par l'arrêt P.A.S. (Il n'en va pas nécessairement ainsi dans tous les cas, du seul fait de l'interdiction expresse d'un acte dans la convention. Encore faudrait-il s'assurer, comme toujours, de l'adéquation de l'offense et de la sanction imposée, de la gravité de l'infraction lorsque la sanction est vraiment de conséquence. Toutefois, il faut convenir que, souvent, l'interdiction d'une conduite donnée dans la convention est une indication de son importance.)

48 Voir supra, p. 359.

49 Re Kingston Independent Nylon Workers Union and Dupont of Canada Ltd, 20 Lab. Arb. Cas. 37 (W.S. Lane, pres.), à la p. $44:$ «... the chairman feels that a very substantial penalty would be justified 》 (en l'occurrence, un congédiement en raison d'un incident créant de l'insécurité, contrairement au règlement interne).

50 Lee v. Compagnie de Téléphone Bell du Canada, supra, note (12), à la p. 186 : «Quant à l'affaire récente de Port Arthur Shipbuilding Co. v. Harry W. Arthur, de la Cour suprême, il faut rappeler que les salariés, en cause, avaient commis un acte expressément prohibé à la convention collective et que cette disposition s'imposait aussi à l'arbitre 》.

51 Re S.K.D. Manufacturing Limited, supra, note (11), à la p. 7, au sujet de l'affaire P.A.S. : «Everyone involved in this case, from the Arbitration. Board up to the Supreme Court, agreed that this was a very serious form of misconduct, exacerbated both by the fact a competitor of the Company was the beneficiary and that the Agreement itself specifically prohibited it $\gg$. 
Nature de la peine.

Dans l'affaire P.A.S., la Cour a conclu à l'excès de pouvoir en raison de l'existence, à ses yeux, d'un motif au congédiement imposé, ou, selon la convention, d'une «juste cause » au congédiement imposé. Raisonnerait-elle nécessairement de la même façon dans l'hypothèse où il s'agirait, non plus d'un congédiement, mais d'une autre sanction? Les pouvoirs de l'arbitre s'en trouveraient-ils alors délimités de la même façon ? Par exemple, devrait-on, par analogie, appliquer l'arrêt P.A.S. dans une situation où un arbitre serait appelé à contrôler l'existence d'un motif d'une suspension?

A cet égard, il faut distinguer l'hypothèse où la peine ne peut varier en sévérité, comme c'est le cas en matière de congédiement, de celle ou elle peut être à «sévérité variable ».

Quand l'employeur exerce le pouvoir de congédier, il ne dispose évidemment que d'un seul mode d'action : il congédie tout simplement. Dans l'affaire P.A.S., on l'a vu, l'arbitre se devait de contrôler le motif de l'employeur en relation avec le congédiement. Rien ne semble s'opposer, logiquement du moins, à ce qu'il fasse de même dans le cas d'une peine à "sévérité invariable ", s'il était saisi de son imposition.

Toutefois, la plupart des autres peines sont, au contraire, de sévérité variable : amende, suspension,... L L'arbitre devrait-il alors se demander si l'employeur avait, tout simplement, un motif à l'imposition d'une amende quelconque ou d'une suspension quelconque ? Ne devrait-il pas chercher à établir s'il était justifié d'imposer une amende d'une quotité déterminée, ou encore, une suspension d'une durée particulière ? La Cour suprême n'a pas répondu à cette question, du moins d'une façon expresse, puisqu'elle ne se posait pas dans l'affaire dont elle était saisie. Par ailleurs, dans l'affaire Int'l Nickel Co. of Canada Ltd, le président du tribunal d'arbitrage a décidé que c'était en relation avec la suspension précise de deux jours infligée en l'occurrence, que le motif de l'employeur devait être contrôlé :

«It is our conclusion, having regard to the provisions of the collective agreement, that the matter before us in this case is whether the twoday suspension imposed upon the grievor was imposed for just cause $\gg 52$.

52 Re United Steelworkers of America and Int'l Nickel of Canada Ltd, supra, note (11), à la p. 121. 
Au seul plan logique, on imagine d'ailleurs difficilement comment on pourrait contrôler l'existence d'un motif valable à suspension, abstraction faite de sa durée; de même dans le cas d'une amende, comment ignorer sa quotité ?

Au strict plan juridiques, cette fois, l'on s'entend pour affirmer que le ratio decidendi d'un cas précédent, partant la solution apportée à ce cas, peut s'appliquer non seulement aux cas subséquents présentant des faits essentiels identiques, ce qui serait extrêmement rare, mais aussi à ceux qui reposent sur des faits de même nature, des faits génériquement semblables. Le problème demeure, évidemment, de savoir jusqu'où peut être poussée la généralisation des faits du précédent.

\section{CONCLUSION.}

En somme, le motif de la sanction étant établi aux yeux de l'arbitre, celui-ci n'a pas à se prononcer sur l'opportunité de la sanction; il ne peut, ni l'annuler, ni y substituer une autre sanction. (Il devra en être de même également, chaque fois que la Cour, appelée à contrôler la décision de l'arbitre, jugera, en se prononçant elle-même abusivement sur le fond, comme dans l'arrêt P.A.S., que l'arbitre aurait dû conclure à l'existence du motif et que, ne l'ayant pas fait, il a commis ce que l'on est convenu d'appeler une «error of law».)

En revanche, en l'absence du motif, d'une «juste cause », selon le langage usuel de la convention, à l'imposition de la sanction, l'arbitre ne se trouvera pas empêché de faire obstacle à la décision de l'employeur ; il pourrait même, dans cette hypothèse, substituer une sanction moins sévère, s'il la jugeait justifiée.

La Cour suprême a voulu, en posant ces limites, rappeler que l'employeur devait avoir ses coudées franches, en matière disciplinaire. Les connaissant, il faudrait maintenant se demander si leur fondement, la prérogative de la direction en matière de discipline, avec plus ou moins de restrictions selon la convention collective, demeure toujours adéquat. L'arbitre joue-t-il le rôle qu'il pourrait jouer, s'il n'était ainsi lié par le résultat des tractations collectives? Ne devrait-il pas, précisément, à l'occasion de ces situations plus importantes qui lui parviennent, pouvoir verser également dans le contrôle de l'opportunité de la sanction, tenir compte, en particulier, de facteurs tels que l'ancienneté, le dossier antérieur du salarié ? 